\title{
An era of safety culture
}

\author{
Subrata Ghosh MD FRCPC FRCP FRCPE
}

C olonoscopy has not only revolutionized the diagnosis and effective management of colonic diseases, especially colorectal neoplasia, but also has been the flag-bearer of quality and safety in endoscopy and in gastroenterology. Well-defined quality and safety parameters have been described, permitting real-time collection of data. The Canadian consensus document published in the current issue of the Canadian Journal of Gastroenterology (pages 71-78) (1) is groundbreaking. A subcommittee of the Canadian Association of Gastroenterology Safety and Quality Indicators in Endoscopy Consensus Group published 19 evidence-based indicators of safety compromise in endoscopy to be recorded by all endoscopy facilities in Canada. Safety and adverse event incidents captured in real time allow for improvement of the entire system, reassure patients and incentivize investment to improve safety, focus training programs and allow incremental measurable gains. Moreover, a real gain is a change in culture in which physicians learn from standardized safety feedback and strive to improve their practice, preferably in a nonpunitive setting.

Colonoscopy has brought about a change in safety culture in the entire field of endoscopic practice. Reporting of adverse incidents in a standardized manner will lead to analysis of contributing factors, understanding of system or individual practitioner issues, distinguish individual incidents from a cluster of events and implement remedial measures expeditiously. The consequences of an adverse incident may be variable - a major incident may not necessarily have a catastrophic outcome, while a minor incident may have a serious outcome. The pressure to work faster and beyond capability, as well as overconfidence and complacency, are cultural issues that adversely affect safety and require careful attention (2). Patients have the right to know the likelihood and consequences of any adverse incident related to procedures to make informed decisions. Communication of an adverse incident to the patient and family is an indispensible skill required from the physician and health organization. The relationship between incident reporting and communication openness can be evaluated in endoscopy practice once we have widespread standardized adverse incident reporting in place (3). A publicly funded health care system is expected to monitor quality and safety in real time, and strive for constant improvement without complacency.

Implementation of adverse event reporting will require leadership, teamwork, ownership, fairness, political will and organizational priorities. There will be challenges in ensuring universal reporting from practices large and small, but the consensus document will permit standardization. In addition, implementation of safety incident reporting in real time in colonoscopy practice should drive similar initiatives in other areas of gastroenterology and hepatology such as inflammatory bowel disease.

The Canadian Journal of Gastroenterology will have a major focus in publishing original research, position papers and consensus statements related to the theme of quality and safety, and this will be a central theme of the philosophy of the Journal. We are aiming to become an international journal in gastroenterology and hepatology, with a major emphasis on quality and safety, often a neglected area of focus in journals, but one that is set to change. We already have a specialist editor who is a leader in the area, and we invite increasing numbers of manuscript submissions related to quality and safety in gastroenterology and hepatology. The publication of the Canadian Association of Gastroenterology Consensus Guidelines on safety and quality indicators in endoscopy (4), as well as the evidence-based indicators of safety compromise (1) in the Journal, should underpin and drive quality improvement in endoscopy and further research into safety and quality in endoscopy. We also invite correspondence related to this area.

\section{BENEFITS OF STANDARDIZED ADVERSE INCIDENT REPORTING AND ANALYSIS \\ 1. Estimate likelihood of an adverse incident. \\ 2. Understand the range of consequences of an adverse incident. \\ 3. Analyze contributing factors related to occurrence of an adverse incident. \\ 4. Monitor the impact of effective implementation of quality and safety measures. \\ 5. Introduce a culture of transparency in quality and safety.}

\section{REFERENCES}

1. Borgaonkar M, Hookey L, Hollingworth R, et al; Canadian Association of Gastroenterology Safety and Quality Indicators in Endoscopy Consensus Group. Can J Gastroenterol 2012;26:71-8.

2. Skevington SM, Langdon JE, Giddins G. 'Skating on thin ice?' Consultant Surgeon's contemporary experience of adverse surgical events. Psychol Health Med 2012;17:1-16.

3. Zwart DLM, Langelaan M, van de Vooren RC, et al. Patient safety culture measurement in general practice. Clinimetric properties of 'SCOPE'. BMC Fam Pract 2011;12:117.

4. Armstrong D, Barkun A, Bridges R, et al. Canadian Association of Gastroenterology consensus guidelines on safety and quality indicators in endoscopy. Can J Gastroenterol 2012;26:17-31.

Department of Medicine, University of Calgary, Calgary, Alberta

Correspondence: Dr Subrata Ghosh, Department of Medicine, University of Calgary, Foothills Medical Centre, North Tower, 1403-29th Street

Northwest, Calgary, Alberta T2N 2T9. Telephone 403-944-8222, fax 403-944-1095, e-mail subrata.ghosh@albertahealthservices.ca Received and accepted for publication January 9, 2012 


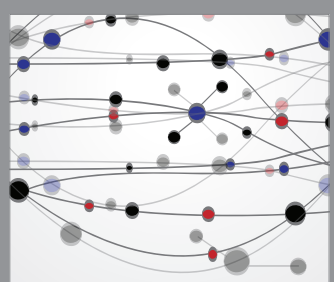

The Scientific World Journal
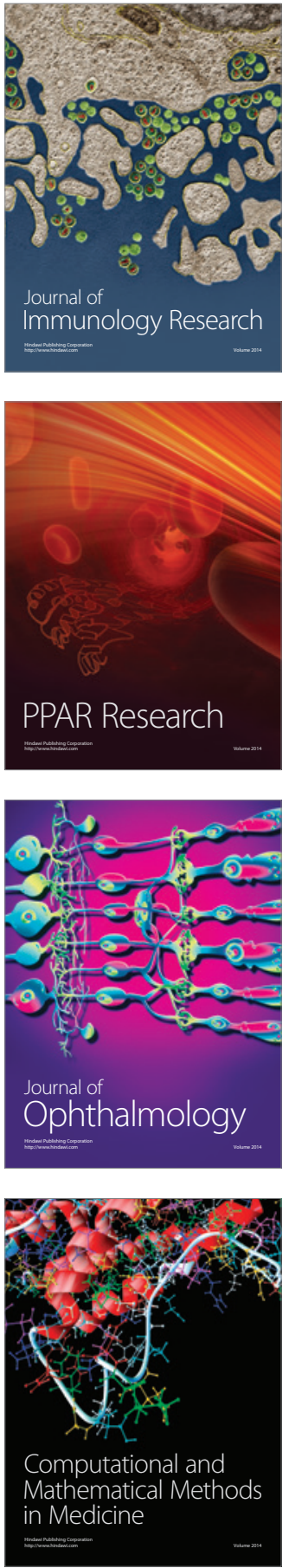

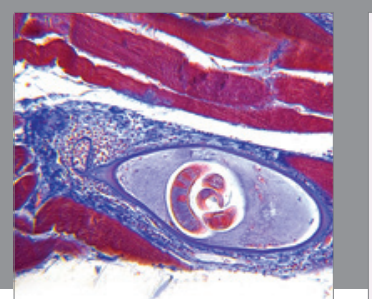

Gastroenterology Research and Practice

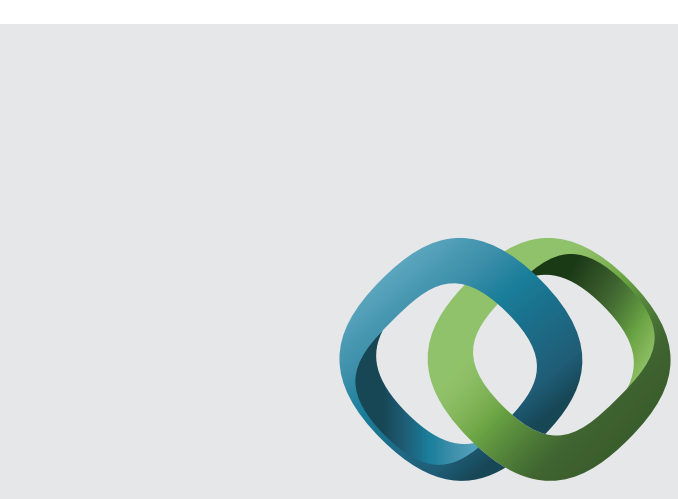

\section{Hindawi}

Submit your manuscripts at

http://www.hindawi.com
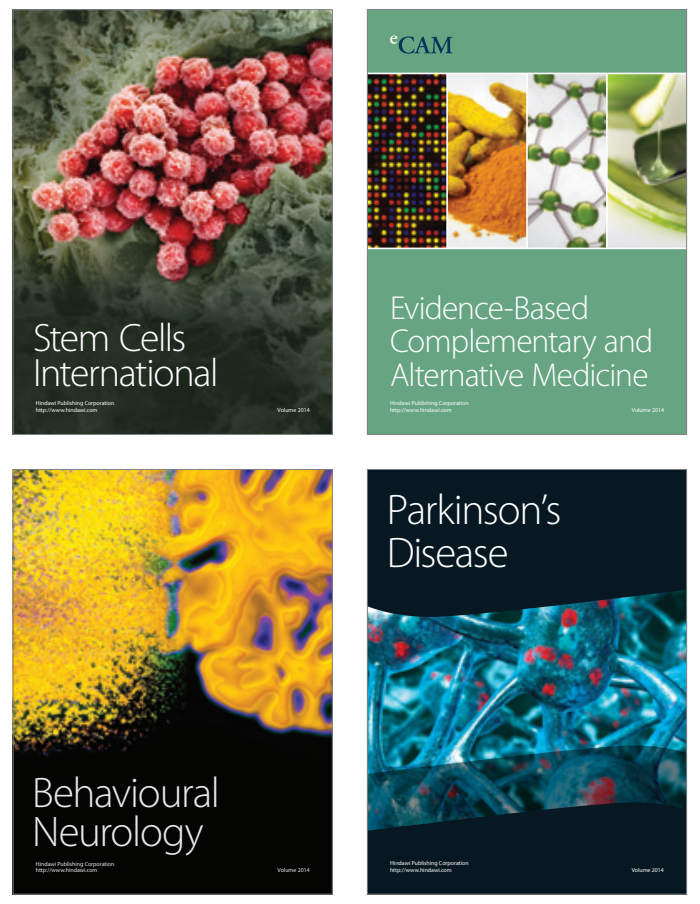
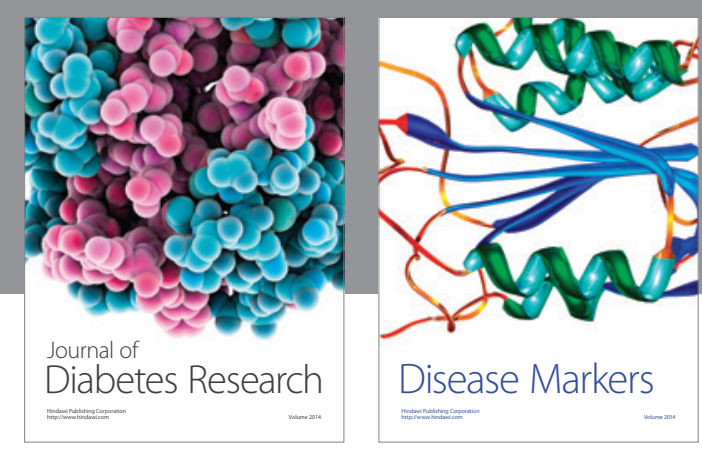

Disease Markers
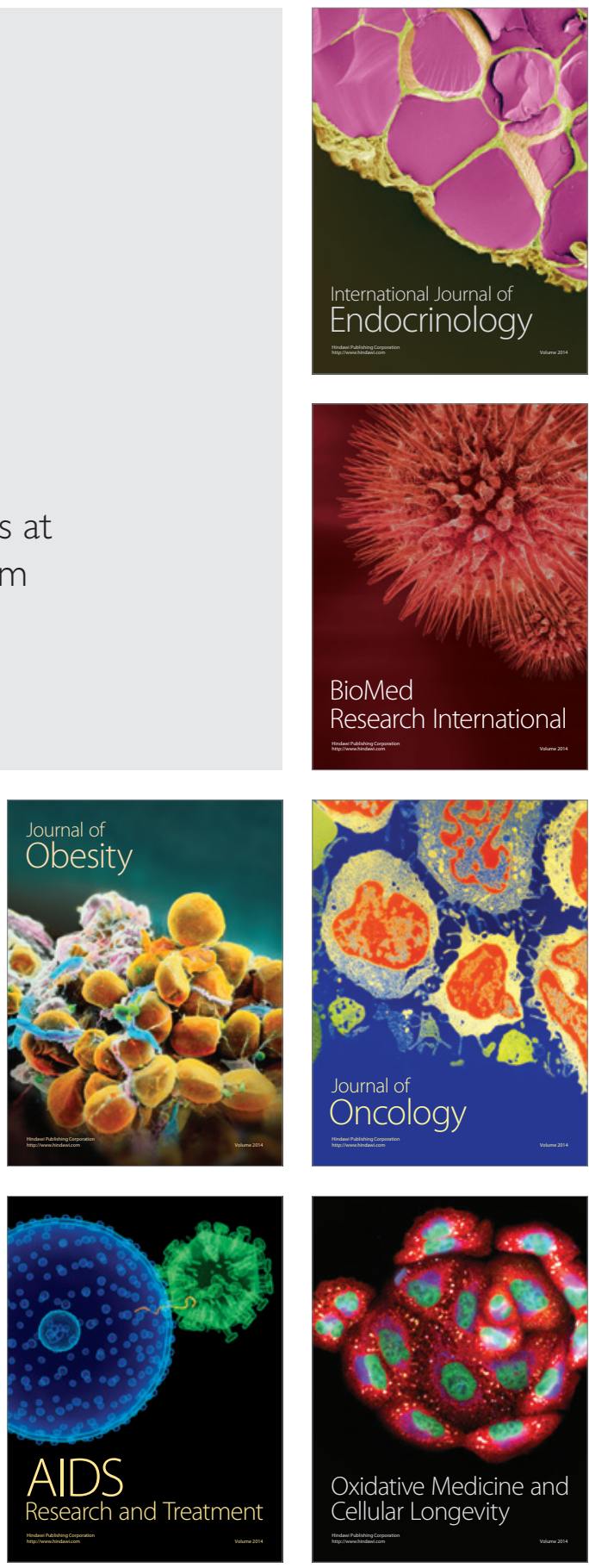